\title{
Pilot study of fascia Bowen therapy for 8- 11 year-old boys with developmental coordination disorder
}

Article

Accepted Version

Creative Commons: Attribution-Noncommercial-No Derivative Works 4.0

Morgan-Jones, M., Knott, F., Wilcox, H. and Ashwin, C. (2019) Pilot study of fascia Bowen therapy for 8-11 year-old boys with developmental coordination disorder. Journal of Bodywork and Movement Therapies, 23 (3). pp. 568-574. ISSN 1360-8592 doi: https://doi.org/10.1016/j.jbmt.2019.02.022 Available at https://centaur.reading.ac.uk/82016/

It is advisable to refer to the publisher's version if you intend to cite from the work. See Guidance on citing.

To link to this article DOI: http://dx.doi.org/10.1016/j.jbmt.2019.02.022

Publisher: Elsevier

All outputs in CentAUR are protected by Intellectual Property Rights law, including copyright law. Copyright and IPR is retained by the creators or other copyright holders. Terms and conditions for use of this material are defined in the End User Agreement.

www.reading.ac.uk/centaur 
Central Archive at the University of Reading

Reading's research outputs online 


\title{
A pilot study of fascia Bowen therapy for 8-11 year-old boys with Developmental Coordination Disorder
}

\author{
Melanie Morgan-Jones ${ }^{1}$, Fiona Knott ${ }^{2}$, Hannah Wilcox ${ }^{3}$ and Chris Ashwin ${ }^{4 *}$, \\ ${ }^{1}$ Department of Health, University of Bath, Bath UK \\ ${ }^{2}$ School of Psychology and Clinical Language Sciences, University of Reading, \\ Reading UK \\ ${ }^{3}$ Department of Clinical Psychology, University of Plymouth, Plymouth UK \\ ${ }^{4}$ Centre for Applied Autism Research, Department of Psychology, University of Bath, \\ Bath UK
}

Author information and qualifications:

MMJ: Dr Melanie Morgan Jones, D Health

FK: Dr Fiona Knott, PhD, DClin

HW: Dr Hannah Wilcox, DClin

CA: Dr Chris Ashwin, PhD

*Corresponding author:

Chris Ashwin,

Centre for Applied Autism Research (CAAR), Dept. of Psychology, University of Bath, Bath UK BA2 7AY

c.ashwin@bath.ac.uk

$+44(0) 1225383502$ 


\begin{abstract}
Background: Developmental Coordination Disorder (DCD), also known as dyspraxia, is characterised by motor skill impairments. The motor difficulties often produce negative effects in other areas of life, such as poor self-esteem and reduced social interactions. One treatment used for DCD is fascia Bowen therapy, which involves stimulating the fascia tissues of the body using finger and thumb rolling movements over the skin to improve overall muscle movement. However, no studies to date have been reported testing the effectiveness of fascia Bowen in DCD.
\end{abstract}

Methods: The present pilot study tested the effectiveness of 6 weeks of fascia Bowen in 10 boys aged 8-11 years with DCD. None of the boys had ever received treatment in any form before this study. Motor skills were assessed using the Movement Assessment Battery for Children-2 (MABC-2) and the DCD questionnaire, and psycho-social functioning was measured using the SelfPerception Profile, Spence Social Skills Questionnaire, and Strengths and Difficulties Questionnaire. All measures of interest were assessed before and after the therapy.

Results: Results showed significant improvement in motor function postintervention, with $60 \%$ of the children no longer clinically being classified as having a movement difficulty on the MABC-2. However, no significant improvements were seen in psycho-social measures.

Conclusions: The current pilot study revealed improvements in motor functioning after fascia Bowen therapy across both performance and questionnaire measures, but improvements did not extend to wider areas. Further research in DCD is needed testing fascia Bowen in larger studies with expanded ages and both genders over longer periods.

Keywords: developmental coordination disorder; dyspraxia; children; fascia Bowen; therapy; motor movement; 


\section{Introduction}

Developmental Coordination Disorder (DCD), also known as dyspraxia, involves a substantial impairment of motor skills compared to what would be expected for a child's age and intelligence level (American Psychiatric Association 2013). DCD is a relatively common disorder of childhood, with prevalence rate estimates of approximately 5\%-6\% for children aged 5-11 (American Psychiatric Association 2013; Leonard 2018). Those with DCD are evident by their general clumsiness and poor posture, and they are slower than normal in their behaviours. Handwriting is usually poor and there are problems in reaching certain developmental milestones during childhood, which often alerts people about potential motor problems.

DCD also impacts other areas of life beyond motor dysfunction, including academic performance, self-esteem, social functioning, family relationships and general wellbeing (Dewey et al 2002; Leonard 2018; Lingam et al 2012). Children with DCD are at risk of developing a variety of psychological and social difficulties, which includes educational under-achievement (Dewey et al 2002; Missiuna et al 2014; Watson \& Knott 2006). These difficulties often emerge because they are slower in completing tasks than their peers and have problems interacting with others across various school settings (Missiuna et al 2006; Zwicker et al 2017).

There are various theories of DCD to explain the underlying mechanisms and these theories have helped inform different approaches to treatment (Blank 2012). The major treatments available for DCD can generally be placed into either bottom-up or top-down theoretical categories, with bottom-up referring to lower-level sensorymotor processing in the body and top-down referring to the higher-level cognitive processes in the brain (World Health Organisation 2001). Bottom-up approaches focus on the sensory-motor deficits in DCD, with atypical sensory-motor processing travelling up to the brain where it exerts effects on the functioning of cortical motor areas. Examples of treatments integrating bottom-up approaches are sensory integration therapy (SIT), process-orientated treatment and perceptual motor training (Mandich et al 2001; Smits-Englesman et al 2013). 
In contrast, top-down approaches emphasise the cognitive and problem-solving processes used to successfully perform tasks (Sugden \& Chambers 1998; Mandich et al 2001; Miller et al 2001; Barnhart et al 2003). Therefore, the focus of these approaches is the cognitive processes within the brain, which then exert their effects on the neural information travelling down to the muscles to perform motor movements. The European Academy of Childhood Disability (EACD) currently recommends the use of top-down approaches for DCD and these guidelines are followed in the UK. This recommendation has been based upon results from metaanalysis studies about treatment effects using motor interventions for children with DCD, which report that task-specific top-down approaches are more effective than bottom-up interventions (e.g. Smits-Engelsman et al 2013). However, available evidence for bottom-up approaches has been weak in terms of the quantity and quality of the research done to date and thus is problematic to interpret (Smits-Engelsman et al 2013). Additionally, there is a genuine lack of transparency in treatment protocols in the field, meaning its often hard to know and interpet the methodologies. The lack of clarity in the literature makes it difficult to determine what approaches are effective or ineffective (Hillier 2007), which creates uncertainty about the quality of evidence investigating bottom-up treatments to date. Therefore, further research testing the efficacy of bottom-up therapies in DCD is needed.

One potential bottom-up intervention for improving DCD is fascia Bowen therapy, which is a derivation of The Bowen procedure that utilises non-invasive light touch movements of a practitioner's fingers and thumbs along different tissue systems of the body including muscles, tendons, ligaments, joints, nerves etc., with the aim to try and re-align the soft tissue structure of the body (Baker 2014; Chaitow 2014b; Wilks \& Knight 2015). Since fascia surrounds all those types of tissues targeted by Bowen techniques, it is inevitable that fascia is affected by the treatment movements, and in different ways to other types of tissues such as muscles (Chaitow 2014a; Wilks \& Knight 2015). Fascia is connected with all tissue types of the body and represents an important network of the body's structure, and dysfunction of this tissue adversely affects many other body systems and abilities (Chaitow 2014a,b; Schleip 2014; Wilks \& Knight 2015). Activity of fascia is a key aspect of body perception, which is an area of function that is compromised in those with DCD (Schoemaker et al 2001). 
Fascia Bowen philosophy believes that the continuity and continuum of the fascia connective tissue in the humans is significant towards optimal functioning (Schleip et al 2012). Fascia Bowen therapy is administered even more lightly than the Bowen movements, by touching the skin so lightly that it does not move the muscles and ligaments as Bowen treatment does. Fascia Bowen is administered in a hoop-like format (following the invisible dermatomal lines of the body) and is designed to more specifically target the fascia soft tissue systems of the body (Chaitow 2014b). Fascia's role begins just below the skin's surface, so its communicative network can be easily manipulated from outside the body, stimulating it to slide hydraulically in an attempt to relax it (Schleip et al 2012). This ability to reduce tension by external stimulation using manual touch is an important feature in a fascia Bowen intervention because anecdotal reports by practitioners note unilateral tightness in children with DCD compared to typically developing peers. Fascia Bowen treatment is desirable because it can be administered directly through clothing, making it is quick and easy for qualified Bowen practitioners to carry out the intervention.

There is evidence that Bowen techniques are effective at improving muscular problems, as well as overall well-being. A systematic review by Hansen \& TaylorPilae (2011) reported that over half the Bowen technique-based studies included in the review were successful in improving a range of different chronic to acute conditions and that some evidence demonstrated reduced symptoms of pain. Dicker (2001) reported about a study of a 6-week Bowen technique treatment in communitydwelling healthcare workers over the age of 41 . Results showed an $89 \%$ improvement in the original symptoms suffered by the participants and that they experienced a $78 \%$ improvement in their ability to work after receiving the Bowen intervention. Results further showed a reduction in stress levels, an improvement in wellbeing and quality of life, including a better ability to sleep and increased energy levels. Other studies of Bowen therapy have shown similar improvements in muscle flexibility and movement, as well as additional areas such as quality of life (Carter 2001, 2002; Dicker 2005; Marr et al 2008). 
While a handful of studies have looked at the effectiveness of The Bowen technique related to the symptoms of specific conditions, no research to date has specifically evaluated the efficacy of fascia Bowen therapy for improving general muscular functioning, as well as psychological and social well-being. The present research aims to fill this gap in the literature by running a preliminary proof of concept pilot-study to test the effectiveness of fascia Bowen therapy for improving motor, psychological, social functioning in a sample of boys with DCD. It was expected that improvements in motor functioning would be seen after therapy compared to before, because the fascia Bowen intervention specifically aims to improve muscular functioning. Given the short time-frame of the 6-week therapy period, it was not known whether psychological, social and behavioural functioning would be higher after therapy compared to before.

\section{Methods}

\subsection{Participants}

The present sample consisted of 10 males aged between 8 and 11 years (mean age $=9.7 ; \mathrm{SD}=1.2)$ recruited from mainstream primary schools across the counties of Berkshire, Oxfordshire and Wiltshire. Information about the study was posted on Facebook sites, shared with dyspraxia groups, and was disseminated through independent occupational therapists (OT's) working in the field. None of the boys had ever had any treatment of any kind for DCD.

All participants had a diagnosis of DCD according to international criteria (APA, 2013), and had scores at or below the 15th centile on the Movement Assessment Battery for Children-2 (MABC-2) as assessed by a registered OT. The 15th centile of the MABC-2 was selected because it is the threshold within the 'Traffic Light' system of the MABC-2 which determines whether a child has a movement difficulty or not. In this system red denotes a significant movement difficulty, amber suggests the child is 'at risk' of having a movement difficulty, and green reflects no detected movement difficulty (see further explanation below). The mean MABC percentile score of participants in the present study was $4.6(\mathrm{SD}=3.53)$, which is in the red area of motor difficulty. 
Exclusion criteria for participation in the study included the presence of co-morbid conditions including hemiplegia and cerebral palsy, or if participants were nonambulant (i.e. unable to walk or move under their own volition). Girls were excluded due to differing developmental milestones between males and females and while differences pre-puberty are moderate, they are more likely to reflect environmental influences such as those which are socially induced by parents, peers, teachers and coaches (Thomas \& French 1985). A further exclusion criterion was that all participants were without intellectual disability, as indicated by a statement of special educational need (or absence thereof). Additionally, none of the participants were receiving any other form of intervention before or during the intervention period.

\subsection{Materials}

\section{Motor functioning: Movement Assessment Battery for Children 2 (MABC-2)}

The MABC-2 (Henderson et al 2007) is designed to be carried out by a qualified professional for the identification and description of impairments in motor performance of children aged 3 to 16 years of age. The MABC-2 includes 8 tasks in total, which produce scores for the three subscales of manual dexterity, ball skills, static and dynamic balance. Scores from all these tasks are converted into centile scores which are incorporated into a 'Traffic light system', which explain a child's total test score in terms of categories of difficulty. Red signifies performance at or below $5^{\text {th }}$ percentile (a significant movement difficulty), amber signifies between the $6^{\text {th }}$ and $15^{\text {th }}$ percentile (the child is 'at risk' of a movement difficulty), and green signifies performance above the $15^{\text {th }}$ percentile (no detected movement difficulty). The MABC-2 shows good reliability and validity (Henderson et al 2007).

\section{The Developmental Coordination Disorder Questionnaire - parent version (DCDQ-P)}

The DCDQ-P (Wilson et al 2009) is completed by the parents about their children's motor performance in everyday activities. It uses a 5-point Likert scale across 15 items, to produce a total score out of 75 . It includes 3 subscales; the subscale 'control during movement' includes 6 items for a score out of 30, 'fine motor and handwriting' includes 4 items for a score out of 20, and 'general co-ordination' has 5 
items for a score out of 25 . Higher scores indicate better motor performance for the child. It is reported to have strong reliability and validity (Wilson et al 2009).

\subsection{Psychological functioning}

\section{Self-Perception Profile for Children (SPP-C)}

The SPP-C (Harter 1985) is a 30-item self-report questionnaire for children to measure self-esteem. It includes five subscales measuring scholastic competence, social acceptance, athletic competence, physical appearance, behavioural conduct and general self-worth. There are 6 items within each of the 5 subscales. The SPP-C uses a 'structured alterative format' whereby each item contains two associated statements, each statement having a choice of two responses each, thereby providing a four-point scale for each item. Scores can fall between 1 and 4 and a mean is calculated, giving a mean score out of a possible 4 for each of the 5 subscales. The SPP-C is reported to have good reliability and validity (Muris et al 2003).

\section{The Self Perception Profile for Children-Parents (SPP-P)}

The SPP-P (Harter 1985) is a parental-report measure of self-esteem which has five subscales covering scholastic competence, social acceptance, athletic competence, physical appearance and behavioural conduct, and one scale assesses overall self-worth. The parent measure is a parallel version to the child version, which was also run in the present research. There are 15 items, with three in each domain. The SPP-P uses a structured alterative format whereby each item contains two associated statements, each statement having a choice of two responses each, thereby providing a four-point scale for each item. Scores can fall between 1 and 4 and a mean is calculated, giving a mean score out of a possible 4 for each of the 5 subscales. Its reliability and validity are reported as acceptable (Boyle et al 2008).

\section{Spence Social Skills Questionnaire-Pupil (SSQ-Pu)}

The SSQ-Pu (Spence 1995) is a 30 item self-report questionnaire measuring social skills using a 3-point Likert-scale. There are 3 subscales measuring conflict resolution/avoidance, warmth and empathy, and social involvement. Participants can 
score up to 20 in each subscale, with a higher score indicating a higher level of social competence. The three subscale scores can be combined to produce a total score. The SSQ-Pu has been shown to have good reliability and internal validity (Spence 1995).

\section{Spence Social Skills Questionnaire-Parent (SSQ-P)}

The SSQ-P (Spence 1995) is a 30-item questionnaire using a 3-point Likert-type rating scale. The item has 3 subscales covering conflict resolution/avoidance, warmth and empathy and social involvement. A score of up to 20 can be achieved for each subscale, with a higher score indicating a higher level of social competence. The three subscale scores can be combined to produce a total score. Its reliability and internal consistency was found to be good with a Guttman split-reliability of 0.90 (Spence 1995).

\section{Spence Social Skills Questionnaire - teacher (SSQ-T)}

Social interaction was also measured using the SSQ-T (Spence 1995), which is completed by the primary teacher and is a parallel version to the child version of the SSQ. The format is the same as in the child version described above. The internal consistency and validity are good, with a Guttman split-half reliability of 0.93 (Spence 1995).

\section{Strengths and Difficulties Questionnaire-Parent (SDQ-P)}

Behaviour was measured by parents using the 25-item SDQ (Goodman 1997), which includes 5 subscales covering emotional symptoms, conduct problems, hyperactivity/inattention, peer relationship problems and pro-social behaviour. Each item has a 3-point Likert scale and each subscale produces a score out of 10. Higher scores indicate a higher level of difficulty, except in the case of the pro-social subscale where a higher score indicates a better level of social functioning. Its reliability and validity are considered to be satisfactory (Hawes \& Dadds 2004).

\section{Strengths and Difficulties Questionnaire - Teacher (SDQ-T)}


The SDQ-T (Hawes and Dadds, 2004) is a teacher-report measure with 25 items in the format of a 3-point Likert-scale. It includes 5 subscales covering emotional symptoms, conduct problems, hyperactivity/inattention, peer relationship problems and pro-social behaviour. Each subscale produces a score out of 10, with the higher score indicating a higher level of difficulty apart from pro-social, where a higher score indicates a better level of social functioning. The reliability and validity are found to be satisfactory (Hawes and Dadds, 2004).

\subsection{Autism co-morbidity}

\section{The Social Communication Questionnaire (SCQ)}

The SCQ (Rutter et al 2003) is a 40-item parent-report measure of Autism Spectrum Disorder (ASD) symptoms and was included here because of high comorbidity between DCD and ASD. The items are administered in a yes/no response format completed by the parent, to produce a score out of 40 . If a child scores 15 or above, then this is highly indicative of ASD. The SCQ has shown excellent sensitivity and specificity for screening of ASD symptomology (Chandler et al 2007).

\subsection{Procedure}

The research involved three different phases, a pre-therapy testing session, a 6week fascia Bowen therapy period and a post-therapy testing session. The study lasted approximately 8-weeks in length for each participant, though one child received his 6 therapy sessions over an 8-week period due to illness and absence from school during that time. The pre- and post-therapy assessments were both carried out by a professionally-qualified OT, as administration of the MABC-2 requires qualification as a medical professional i.e. a doctor, nurse, or OT. The OT in this study regularly used the MACB-2 as part of their standard practice, and was already very familiar with using it. The OT was aware that all the boys were receiving the fascia Bowen treatment because of the lack of a control group. However, the OT had no knowledge about fascia Bowen treatment or any experience with it.

\section{Pre-Therapy Assessments}


The pre-therapy motor assessments took place in schools for 9 of the participants and a 10th participant was assessed at home at the request of his parents. For this an OT completed the MABC-2 with each of the children to assess initial motor functioning. The children also completed the SPP-C and SSQ-P, and an appropriate member of staff at each child's school completed the SSQ-T and the SDQ-T. The SSQ-P, DCDQ-P, SPP-P Children, SDQ-P, and the SCQ were completed with a parent of each child via telephone.

\subsection{Fascia Bowen therapy}

The fascia Bowen therapy took place during weeks 2-7 of the study for all of the children, apart from one who completed it during weeks 2-9 due to illness during that period affecting two of the weeks. Each individual therapy session took approximately 45 minutes and each participant received one session per week for a total of 6 weeks. Sessions took place in a private room in the child's school (7 participants) or in a non-NHS clinic (3 participants), depending on which option was most convenient for the participant and their family. Each child was brought into the treatment room by a teacher or a parent and the child was asked politely to lie prone and fully clothed on the treatment couch. The child was then asked if they were comfortable and told to let the practitioner know if they felt uncomfortable at any point during the session. Once they were comfortable and ready, the treatment was begun.

The fascia Bowen therapy was carried out to target movement of the fascia only, and involved taking the 'skin slack' and moving it back and then forward again to release the skin. This was done without specifically making the rolling moves over the muscles and ligaments that are characteristic of Bowen therapy. Instead, each fascia Bowen move is done at the level of the superficial fascia with the aim to target the relationship between the fascia and the nerves, tendons or muscles in each area being focused upon. These were carried out using short sequences at key structural points of the body by applying the movements in a hoop-like direction to follow the invisible dermatomal lines of the body. Each sequence of moves focused on a key area of the body, and then was followed by a short break lasting approximately a couple of minutes. The breaks are included to allow the body to rest for a few minutes in order to initiate the processes of repair. Using these types of movements, a 
standardised treatment protocol regime was followed that was originally devised by Howard Plummer, which involves moving from one area to another across the body following the dermatomal lines. Further information about the specifics steps of the therapy are available in a standardized teaching manual, however this manual is not currently available in the public domain. The steps used in the present research involved those generally followed by professional practitioners who have been trained to administer fascia Bowen therapy.

The whole treatment protocol was administered over a period of 45 minutes, with the children receiving treatment lying in both prone and supine positions during the session according to the fascia Bowen protocol instruction sheet. The practitioner did not engage in general conversation with the child during the treatment process. At the end of the treatment protocol the child was asked to come down from the treatment couch and he was then escorted to the door where he was met by his teacher or a parent, depending upon where the treatment had taken place.

\section{Post-therapy Assessments}

In week 8 of the research the same measures were carried out as in the pre-therapy with the children, teachers and parents. For one of the children this occurred during week 10.

\section{Results}

The mean SCQ score for the current sample was $13.6(\mathrm{SD}=4.79)$, which is below the cut-off score of 15 which suggests possible ASD (Rutter et al 2003). In addition, 7 out of the 10 children had individual SCQ scores below the cut-off score of 15 . The outcome data of the 3 children who met the screening threshold was compared to the data for the other 7 children, and little or no difference was seen in the scores between them on any measure. This included the MABC- 2 data, as the 3 participants who scored above 15 were not the ones who showed the least or the most change in motor functioning following the therapy.

\subsection{Motor functioning}

\section{MABC-2 results}


A MANOVA was conducted with the 3 MABC-2 subscales (manual dexterity, aiming and catching, balance) as the DV's and time (pre-therapy and post-therapy) as the IV. Results of the MANOVA revealed that time had a significant effect on the MABC-2 scores, $F(3,7)=7.44, \mathrm{p}=.014, \eta^{2}=.76$ (see Figure 1). Follow up univariate analyses within the three subscales were therefore conducted. The times for manual dexterity score were significantly different between time 1 and $2, F(1,9)=6.1, \mathrm{p}=$ $\left.0.04, \eta^{2}=0.4\right)$, with scores being higher post-intervention $(\mathrm{M}=15, \mathrm{SE}=4.75)$ compared to pre-intervention ( $\mathrm{M}=4.15, \mathrm{SE}=1.02)$. The times for aiming and catching were also significantly different between the times, $F(1,9)=8.3, \mathrm{p}=.01, \eta^{2}=0.48$, with scores being higher post-intervention $(\mathrm{M}=26.85, \mathrm{SE}=7.01)$ compared to pre-intervention $(\mathrm{M}=11.4, \mathrm{SE}=3.78)$. The times for balance scores were once again significantly different from pre- to post-therapy, $F(1,9)=14.6, \mathrm{p}=.0004, \eta^{2}=0.62$, with scores being higher post-intervention $(\mathrm{M}=55.1, \mathrm{SE}=10.07)$ compared to pre-intervention $(\mathrm{M}=23, \mathrm{SE}=6.79)$.

\section{PLACE FIGURE 1 HERE}

The mean total percentile score showed that the pre-intervention total was in the red zone (Mean=4.6 $\leq 5$ th percentile; see Table 1). The mean post total percentile score showed that the post-intervention total was in the green zone (Mean=23.5 $\geq$ 15 th percentile). Looking individually at the total percentile score for each child, table 1 demonstrates how many children moved between categories on their movement difficulties. It was found that 3 children remained in the red category, 1 child moved up one category from red to amber, 4 children moved up 2 categories from red to green and 1 child moved up one category from amber to green. This means that 6 children, post-intervention, were now in the category classed as no longer having a movement difficulty.

\section{DCDQ-P results}


A MANOVA was conducted with the mean scores for the 3 subscales of the DCDQ-P (control movement, handwriting, co-ordination) as the DV's and time (pretherapy and post-therapy) as the IV. Results using Pillai's trace showed there was a trend towards a significant effect of time on the subscales of the DCDQ-P, $F(3,7)=$ $3.33, p=.08, \eta^{2}=.59$ (see Figure 2). Follow-up tests of univariate effects showed there was a significant effect of control movement, $F(1,9)=10.8, \mathrm{p}=.01, \eta^{2}=.54$, with scores at post-therapy $(\mathrm{M}=19.5, \mathrm{SE}=1.19)$ higher than those at pre-therapy $(\mathrm{M}=15.5, \mathrm{SE} .872)$. There was no significant effect of time for the coordination scores, $F(1,9)=1.33, \mathrm{p}=.28, \eta 2=.13$, nor was there a significant effect of time for handwriting scores, $F(1,9)=.19, \mathrm{p}=.67, \eta^{2}=0.02$.

\section{PLACE FIGURE 2 HERE}

\section{Psychological functioning results}

A mixed MANOVA was conducted with time (pre-therapy versus post-therapy) and respondent (parent versus pupil) as the IV's and all 5 subscales of the SelfPerception Profile (SPP) (scholastic competence, social acceptance, athletic competence, physical appearance, behavioural conduct) as the DV's. The results of the MANOVA showed there was no significant effect of time on the mean SPP scores, $\left.\mathrm{F}(5,5)=2.45, \mathrm{p}=.17, \eta^{2}=.71\right)$. The MANOVA further showed, using Pillai's trace, that there was a significant main effect of respondent on the SPP scores, $F(5,5)=6.92, \mathrm{p}=.027, \eta^{2}=.8$. Follow-up tests of univariate effects showed there was a significant effect of respondent on the athletic competence subscale, $F(1,9)=24.3$, p $=.001$, with pupils rating higher $(\mathrm{M}=2.4, \mathrm{SE}=.19)$ than parents $(\mathrm{M}=1.66, \mathrm{SE}=.22)$ on athletic competence also parents rating higher $(\mathrm{M}=3.87, \mathrm{SE}=.06)$ than pupils $(\mathrm{M}=3.03, \mathrm{SE}=1.4)$ on physical appearance. There were no significant differences in the remaining scales (scholastic competence, social acceptance, behavioural conduct). There was no significant multivariate interaction between time and respondent $F(5,5)=2.64, \mathrm{p}=.15, \eta^{2}=.73$. 


\section{Social functioning results}

A 2-by-3 mixed ANOVA was conducted on the total SSQ scores with time (pretherapy versus post-therapy) and respondent (parent versus pupil versus teacher) as the IV's. The ANOVA showed there was no main effect of time, $F(1,9)=2.82$, p = $.127, \eta^{2}=.24$ (pre therapy score $=42.6, \mathrm{SD}=2.6$; post therapy score $=39.8, \mathrm{SD}=3.2$ ). The results further showed a trend towards a significant main effect of respondent, $\left.F(2,18)=3.07, \mathrm{p}=.07, \eta^{2}=.25\right)$, with teacher scores $(44.6, \mathrm{SD}=3.8)$ lower than both the parents' $(42.5, \mathrm{SD}=3.1)$ and children's scores $(44.6, \mathrm{SD}=1.8)$. The ANOVA also showed that there was no significant interaction between respondent and time, $F(2,18)=.31, \mathrm{p}=.74, \eta^{2}=.03$.

\section{Behavioural functioning}

A mixed ANOVA on the total scores of the difficulty scales of the SDQ was conducted with Time (pre-therapy versus post-therapy) and respondent (teacher versus parent) as the IV's. Results showed there was no significant main effect of respondent, $\mathrm{F}(1,9)=2.085, \mathrm{p}=.709, \eta^{2}=0.016$ (parent report $=17.1, \mathrm{SD}=1.47$; teacher report $=14.6, \mathrm{SD}=1.6$ ), nor a significant main effect of time, $F(1,9)=.48, \mathrm{p}=$ $.57, \eta^{2}=0.05$ (pre therapy score $=16.3, \mathrm{SD}=1.7$; post therapy score $=15.3, \mathrm{SD}=$ 1.45). The ANOVA also showed there was no significant interaction between time and respondent, $F(1,9)=.148, \mathrm{p}=0.709, \eta^{2}=0.05$.

A separate mixed ANOVA was conducted on the SDQ pro-social subscale scores with respondents (teacher versus parent) and time (pre-therapy versus post-therapy) as the IV's. This was done because as the SDQ manual states that the scores for this subscale should not be combined with the other SDQ scales. The results from the mixed ANOVA showed that the difference between respondents was non-significant, $F(1,9)=1.29, \mathrm{p}=.285$, and the effect size $\left(\eta^{2}=.126\right)$ was small (see Figure 3). Similarly, there was no significant effect of time, $F(1,9)=3.15, p=.109, \eta^{2}=.260$. ANOVA showed that there was a significant interaction between respondent and time on pro-social SDQ score, $F(1,9)=5.19, p=.049, \eta^{2}=.366$. A post-hoc pairedsamples T-test was conducted to examine the difference between the Teacher scores only which showed they were higher pre-intervention $(\mathrm{M}=6.5, \mathrm{SD}=2.84)$ compared to post-intervention $(\mathrm{M}=2.75, \mathrm{SE}=.22), \mathrm{t}(9)=2.75, \mathrm{p}=.022$. 


\section{PLACE FIGURE 3 HERE}

\section{Discussion}

The aim of this pilot study was to investigate if a 6-week fascia Bowen therapy improved motor functioning, as well as psychological, social and behavioural functioning, in a sample of boys aged 8-11 diagnosed with DCD. The results showed that motor functioning was improved in the sample of boys with DCD after the fascia Bowen therapy compared with before, such that most of the boys no longer met the motor impairment criteria for DCD after receiving the therapy. However, no improvements were seen in psychological, social or behavioural functioning after the therapy compared to before. Therefore, while the current findings showed that a 6week fascia Bowen therapy improved muscular functioning in a small group of boys with DCD, these improvements did not extend to further areas of life.

Significant improvement on the MABC-2 scores of the children with DCD was demonstrated from before to after the fascia Bowen therapy sessions. The improvements were seen across all the subscales of the MABC-2, including manual dexterity, aiming, and catching and balance scores. After the study, six of the 10 participating children no longer fell into the category of having a movement difficulty according to their MABC-2 "traffic light" system, and another child showed improvement that resulted in being labelled only in the "at risk" category of movement difficulty after the therapy. The motor improvements generally involved medium to large effect sizes, which was surprising and promising given that previous literature has often not reported strong effect sizes for bottom-up interventions (Novak 2013; Smits-Engelsman et al 2013). Together, the present research provides initial evidence for the efficacy of fascia Bowen therapy for improving movement difficulties in children with DCD, although further research in this area including control groups is needed.

The improvement in the children's muscular functioning after the fascia Bowen intervention involved improved manual dexterity, aiming and catching and balance. The present results are consistent with previous studies showing that Bowen therapy improves motor functioning in other specific conditions and groups (Hansen \& 
Taylor-Pilae 2011), and extends the findings to include DCD. The muscular improvements may translate to better performance in everyday activities for children with DCD, such as reduced clumsiness in the form of falling over and colliding with obstacles, less dropping of objects, and greater chance to participate in games with other children involving ball skills. However, the current results of improved muscular functioning from fascia Bowen therapy did not also translate to improvements in psychological, social and behavioural domains, or in scholastic functioning. The lack of increases in self-esteem, social interaction and behavioural strengths after the therapy may show that fascia Bowen only targets muscular issues in DCD, though in itself this means the therapy is effective in improving the key muscular problems characteristic of DCD. Another explanation for the lack of generalisation of improvements to other areas could also be due to the relatively short 6-week time period of the therapy in the present research for evaluating improvements in psycho-social and scholastic functioning, which may need to be evaluated over many months. Further research is needed to evaluate if improved muscular functioning from fascia Bowen therapy translates into improvements in social, psychological and behavioural areas of life over longer time periods of testing.

\subsection{Limitations}

A limitation of the present research was that it only included 10 boys with DCD, with no control group included in the study for comparison purposes. The small sample was due to the general difficulties in recruiting sufficient numbers of participants with DCD for therapy purposes, and only males were included because there is a higher prevalence rate of males than females in DCD. Therefore, the results need to be replicated with the inclusion of a matched control group for comparisons, which either does not receive treatment or receives an alternative treatment. The replication should also include larger samples of participants with DCD, which should include both boys and girls. Since only one experienced OT assessed all the participants in the present study, there may have been biases in the results due to the familiarity of the participants with the OT and because the OT was aware of the nature of the study. Future research should include larger studies with expanded ages and both genders, as well as procedures to make the OT blind to diagnosis or group. Despite these limitations, the present study has helped provide proof of concept about fascia 
Bowen therapy in DCD as effective in improving the muscular ability of children with DCD. 
Acknowledgements: We would like to thank the participants who participated and their families and teachers for helping with the research.

Funding: This research did not receive any specific grant from funding agencies in the public, commercial, or not-for-profit sectors.

Conflicts of interest: none. 


\section{References}

American Psychiatric Association 2013 Diagnostic and Statistical Manual of Mental Disorders (Fifth ed.). American Psychiatric Publishing, Arlington.

Baker J 2014 Bowen Unravelled: A Journey into the Fascial Understanding of the Bowen Technique. North Atlantic Books, Berkeley.

Barnhart RC, Davenport MJ, Epps SB, Nordquist VM 2003 Developmental Coordination Disorder. Journal of the American Physical Therapy Association 83: 722-731.

Blank R 2012 European Academy of Childhood Disability (EACD):

Recommendations on the definition, diagnosis and intervention of developmental coordination disorder. Developmental Medicine and Child Neurology 54: 14698749.

Boyle GJ, Matthews G, Saklofske DH 2008 The SAGE Handbook of Personality Theory and Assessment: Personality Theories and Models, SAGE Publications.

Carter B 2001 A pilot study to evaluate the effectiveness of Bowen technique in the management of clients with frozen shoulder. Complementary Therapies in Medicine 9: 208-215.

Carter B 2002 Clients' experiences of frozen shoulder and its treatment with Bowen technique. Complementary Therapies in Nursery and Midwifery 8: 204210.

Chaitow L 2014a Fascia research and clinical applications in prevention and treatment of pain and dysfunction. The British Fascia Symposium. Windsor.

Chaitow L 2014b Fascial Dysfunction $\left(1^{\text {st }}\right.$ Ed). Handspring Publishing Limited, Encaitland.

Chandler S, Charman T, Baird G, Siminoff, Loucas, T, Meldrum D, Scott M, Pickles A 2007 Validation of the Social Communication Questionnaire in a Population Cohort of Children with Autism Spectrum Disorders. Journal of the American Academy of Child and Adolescent Psychiatry 46: 1324-1332.

Dewey D, Kaplan BJ, Crawford SG, Wilson BN 2002 Developmental coordination disorder: Associated problems in attention, learning, and psychosocial adjustment. Human Movement Science 21: 905-918.

Dicker A 2001 Using Bowen Therapy to improve staff health. Australian Journal of Holistic Nursing 8: 38-42.

Dicker A 2005 Bowen technique--its use in work related injuries. Australian Journal of Holistic Nursing 12: 31-34.

Goodman R 1997 The Strengths and Difficulties Questionnaire: A Research Note. Journal of Child Psychology and Psychiatry 38: 581-586.

Hansen C, Taylor-Piliae RE 2011 What is Bowenwork ${ }^{(\mathrm{R})}$ ? A systematic review. Journal of Alternative and Complementary Medicine 17: 1001-1006.

Harter S 1985 The Self-Perception Profile for Children. University of Denver, Denver.

Hawes DJ, Dadds MR 2004 Australian data and psychometric properties of the Strengths and Difficulties Questionnaire. Australian and New Zealand Journal of Psychiatry 38: 644-651.

Henderson SES, Sugden DA, Barnett AL 2007 Movement Assessment Battery for Children - 2 Examiner's Manual. London: Harcourt Assessment.

Hillier S 2007 Intervention for Children with Developmental Coordination Disorder: A Systematic Review. The Internet Journal of Allied Health Sciences and Practice 5: $1540-1580$.

Leonard HC 2018 Developmental Coordination Disorder. In E. Braaten (Ed) 
Encyclopedia of Intellectual and Developmental Disorders. SAGE Publications, London.

Lingam R, Jongmans MJ, Ellis M, Hunt LP, Golding J, Emond A 2012 Mental health difficulties in children with developmental coordination disorder. Pediatrics 129: e882-891.

Mandich AD, Polatajko HJ, Macnab JJ, Miller LT 2001 Treatment of children with Developmental Coordination Disorder: What is the evidence? Physical and Occupational Therapy in Pediatrics 20: 51-68.

Marr M, Lambon N, Baker J 2008 Effects of the Bowen Technique on flexibility levels: Implications for facial plasticity. Journal of Bodywork and Movement Therapy 12: 388.

Miller LT, Polatajko HJ, Missiuna C, Mandich AD, Macnab JJ 2001 A pilot trial of a cognitive treatment for children with developmental coordination disorder. Human Movement Science 20: 183-210.

Missiuna C, Gaines R, Soucie H, Mclean J 2006 Parental questions about developmental coordination disorder: A synopsis of current evidence. Paediatrics and Child Health 11: 507-512.

Missiuna C, Cairney J, Pollock N, Campbell W, Russell DJ, Macdonald K, Schmidt L, Heath N, Veldhuizen S, Cousins M 2014 Psychological distress in children with developmental coordination disorder and attention-deficit hyperactivity disorder. Research in Developmental Disabilities 35: 1198-1207.

Muris P, Meesters C, Fijen P 2003 The Self-Perception Profile for Children: Further evidence for its factor structure, reliability, and validity. Personality and Individual Differences 35 (8): 1791-1802.

Novak I 2013 Evidence to Practice Commentary New Evidence in Developmental Coordination Disorder (DCD). Physical and Occupational Therapies in Pediatrics 33: 170-173.

Rutter M, Bailey A, Lord C 2003 The social communication questionnaire: manual. Los Angeles, USA: Western Psychological Services.

Schleip R 2014 Fascia as sensory organ. The British Fascia Symposium. Windsor.

Schleip R, Findley TW, Chaitow L, Huijing PA 2012 Fascia: The Tensional Network of the Human Body: The Science and Clinical Applications in Manual and Movement Therapy ( $1^{\text {st }}$ Ed.). Churchill Livingstone Elsevier, London.

Schoemaker MM, Van der Wees M, Flapper B, Verheij-Jansen N, Scholten-Jaegers S, Geuze RH 2001 Perceptual skills of children with developmental coordination disorder. Human Movement Science 20: 111-133.

Smits-Engelsman BC, Blank R, Van der Kaay AC, Mosterd-van der Meijs R, Vlugt-van den Brand E, Polatajko HJ, Wilson PH 2013 Efficacy of interventions to improve motor performance in children with developmental coordination disorder: a combined systematic review and meta-analysis. Developmental Medicine and Child Neurology 55: 229-237.

Spence SH 1995 Social Skills Training: Enhancing Social Competence with children and Adolescents. Windsor, UK: NFER-Nelson.

Sugden DA, Chambers ME 1998 Intervention approaches and children with developmental coordination disorder. Pediatric Rehabilitation 2: 139-147.

Thomas JR, French KE 1985 Gender differences across age in motor performance a meta-analysis. Psychological Bulletin 98: 260-282.

Watson L, Knott F 2006 Self-esteem and coping in children with developmental coordination disorder. British Journal of Occupational Therapy 69: 450-456. 
Wilks J, Knight I 2015 Using the Bowen Technique to Address Complex and Common Conditions. Singing Dragon, London.

Wilson BN, Crawford SG, Green D, Roberts G, Aylott A Kaplan BJ 2009 Psychometric properties of the revised Developmental Coordination Disorder Questionnaire. Physical and Occupational Therapy in Pediatrics 29: 182-202.

World Health Organisation 2001 International Classification of Functioning, Disability and Health. World Health Organisation, Geneva.

Zwicker JG, Suto M, Harris SR, Vlasakova N, Missiuna C 2017 Developmental coordination disorder is more than a motor problem: Children describe the impact of daily struggles on their quality of life. British Journal of Occupational Therapy 81: 65-73. 
Table 1.

Pre- and post-intervention centile category for each participant in the Movement Assessment Battery for Children 2, with higher scores reflecting better motor ability. Red denotes motor performance at or below the $5^{\text {th }}$ percentile (significant movement difficulty), amber signifies motor performance between the $6^{\text {th }}$ and $15^{\text {th }}$ percentile (at risk of a movement difficulty), and green denotes motor performance above the $15^{\text {th }}$ percentile (no detected movement difficulties).

\begin{tabular}{|l|c|c|}
\hline Participant & $\begin{array}{c}\text { Pre-Intervention } \\
\text { centile }\end{array}$ & $\begin{array}{c}\text { Post-Intervention } \\
\text { centile }\end{array}$ \\
\hline 1 & 1 & 5 \\
\hline 2 & 9 & 63 \\
\hline 3 & 0.5 & 5 \\
\hline 4 & 5 & 16 \\
\hline 5 & 2 & 9 \\
\hline 6 & 0.5 & 1 \\
\hline 7 & 9 & 37 \\
\hline 8 & 5 & 37 \\
\hline 9 & 9 & 25 \\
\hline 10 & 5 & 37 \\
\hline
\end{tabular}


Figure Captions

Fig. 1. Graph showing mean scores for the Movement Assessment Battery for Children version 2 (MABC-2) subscales across the pre- and post-intervention times, with higher scores reflecting better motor functioning. Note the error bars represent standard error of the means.

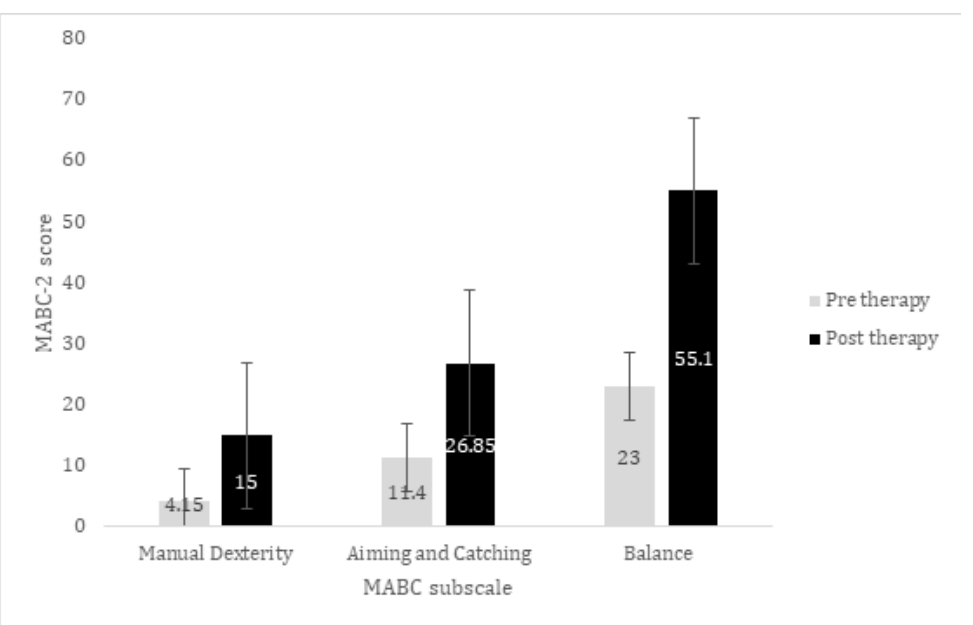

Fig. 2. Graph showing the mean scores pre- and post-intervention for the Developmental Coordination Disorder Questionnaire - parent version, with higher scores indicating better motor performance. Note the error bars represent standard error of the means.

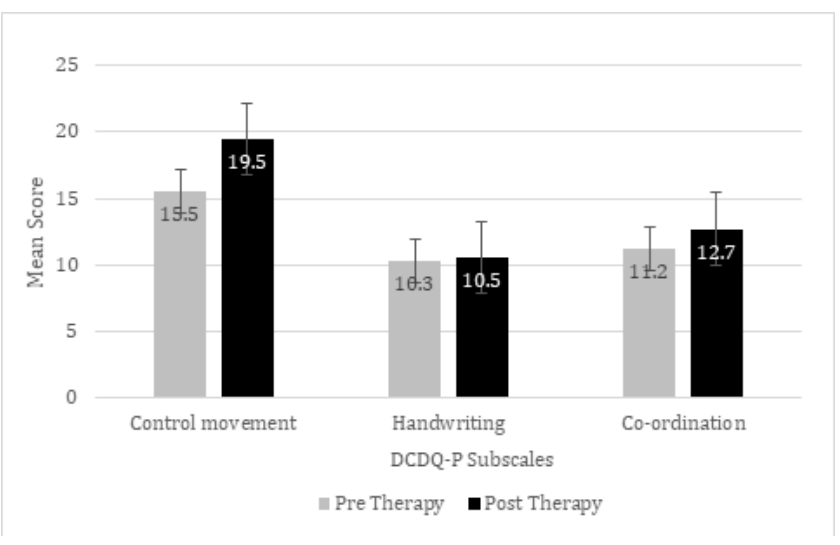


Fig. 3. Graph showing mean scores at pre- and post-intervention for the prosocial scale of the Strengths and Difficulties Questionnaire (teacher and parent versions), with higher scores indicating higher levels of prosocial behaviour. Note the error bars represent standard error of the means.

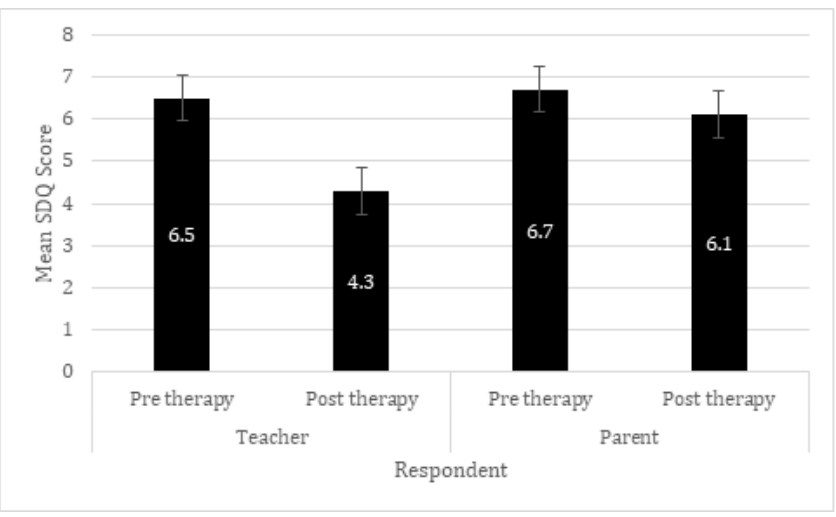

